\title{
Long noncoding RNA LINC005 I I promotes cell growth and invasion in triple-negative breast cancer by interacting with Snail
}

This article was published in the following Dove Press journal: Cancer Management and Research

\author{
Ruilei Liu',* \\ Liang Wang ${ }^{2, *}$ \\ Tianyu Gan' \\ Tao $\operatorname{Pan}^{3}$ \\ Jianglong Huang ${ }^{4}$ \\ Mingjun $\mathrm{Bai}^{3}$
}

'Department of Thyroid and Breast Surgery, The Third Affiliated Hospital, Sun Yat-sen University, Guangzhou, People's Republic of China; ${ }^{2}$ Department of Gastrointestinal Surgery, The First Affiliated Hospital, Sun Yat-sen University, Guangzhou, People's Republic of China; ${ }^{3}$ Department of Vascular Interventional Radiology, The Third Affiliated Hospital, Sun Yat-sen University, Guangzhou, People's Republic of China; ${ }^{4}$ Department of Gastrointestinal Surgery, The Third Affiliated Hospital of Sun Yat-sen University, Guangzhou, People's B of China

*These authors contribut equally to this work

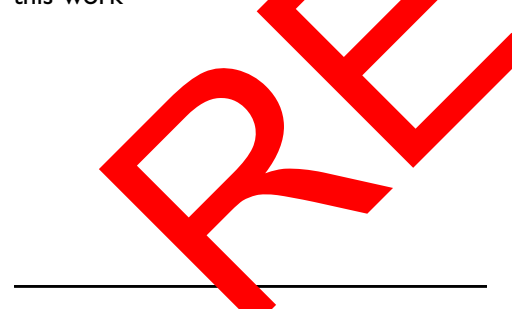

Correspondence: Jianglong Huang Department of Gastrointestinal Surgery, The Third Affiliated Hospital of Sun Yatsen University, 600 Tianhe Road, Guangzhou, People's Republic of China Email baimingj@mail.sysu.edu.cn

Mingjun Bai

Department of Vascular Interventional Radiology, The Third Affiliated Hospital, Sun Yat-sen University, 600 Tianhe Road, Guangzhou, People's Republic of China Email drhjl@aliyun.com
Purpose: Aberrant long noncoding RNA express has been free nt/ eported in cancer research, including in triple-negative breast cance $\mathrm{NBC}$ he aim of the present study was to investigate the involvement of LINC00511 a he pro sion and gnosis of TNBC.

Materials and methods: The express tevel of LIN 05 was examined by RT-PCR in TNBC tissues and in cell lines. MTP and ca ny formationassays were used to examine the cell growth ability. A Boyden assav was used to vamine the cell invasion ability. RNA pulldown and RNA immunopre pitation (RIP) assay vere used to examine the proteins that interacted with LINC0051

Results: We demonstrate that the LIN 00511 expression level was elevated in TNBC tissues when comnared with in norm breast tissues. The downregulation of LINC00511 decreased TNBC tenth and invasion compared to those of the controls. To explore the molecular mechat ms ang the biological activity of LINC00511, we identified protein bound LINC00511 with RNA pull-down experiments. We showed that LIN 0511 inds to the $\beta$-transducin repeat containing (BTRC) E3 ubiquitin protein. chanisti Iv INC 511 maintained the stability of Snail by impeding its ubiquitination and ation by me BTRC E3 ubiquitin protein.

Conclu on: Our data suggested that LINC00511 might serve as a novel molecular target for the trea ent of TNBC.

ywords: triple-negative breast cancer, LINC00511, Snail

\section{Plain language summary}

Our study aimed to examine the biological role and the underlying mechanism of LINC00511 in TNBC. We demonstrated that LINC00511 expression levels were elevated in TNBC tissues compared to those in the controls. The inhibition of LINC00511 decreased TNBC cell growth and invasion compared to those of the controls. In addition, the downregulation of LINC00511 reversed the epithelial-mesenchymal transition (EMT) phenotype. Mechanistically, LINC00511 maintained the stability of Snail by impeding its ubiquitination and degradation via the BTRC E3 ubiquitin protein. We propose that the LINC00511/Snail axis may be a useful molecular target for the treatment of TNBC.

\section{Introduction}

Breast cancer, with its high incidence rate over the past decades, is the most common type of cancer and the second most frequent cause of cancer-related mortality among women. ${ }^{1}$ During the earliest stages of tumor development, breast 
cancer cells usually enter the circulation, therefore resulting in the formation of metastatic lesions, which are responsible for $\sim 90 \%$ of the breast cancer-related mortality. ${ }^{2,3}$ Triple-negative breast cancer (TNBC), a subtype of breast cancer, does not grow in response to the hormones estrogen, progesterone, or HER2/neu. ${ }^{4}$ TNBC characteristically has a higher grade than that of other breast cancer subtypes and tends to metastasize. The five-year survival rate for TNBC is approximately $77 \%$ vs the rate of $93 \%$ that is observed for other breast cancer types. ${ }^{5}$ The molecular mechanisms of tumorigenesis in TNBC are still not fully understood. Therefore, it is an urgent need to elucidate the molecular mechanisms underlying breast cancer processes.

Long noncoding RNAs (lncRNAs), a class of noncoding RNAs that are over 200 nucleotides in length, play a significant role in a series of biological processes. ${ }^{6}$ Recently, studies have reported that the dysregulation of lncRNAs is involved in several pathological states, including cancer. ${ }^{7}$ LncRNAs exert their function by affecting chromatin remodeling and transcriptional and posttranscriptional regulation.

Snail is a key regulator of the epithelial-mesenchymal transition (EMT) of tumor cells. Mechanistically, Snail o repress the transcription of the cell adhesion molecul E-cadherin and ultimately contributes to the EMT phenotype. ${ }^{8}$ The elevated expression of $\mathrm{S}$ has peen observed in a series of cancers, including NBC..$^{9}$

In the present study, we explored ne $\lambda_{\text {sion and }}$ role of LINC00511 in breast cance 7 addition, investigated the interaction of LINC 0511 d Snail in oreast cancer cells. Our findings $r$ ealed a nov nechanism of LINC00511/Snail intera on, which promoted cell growth and invasion in breast ncer

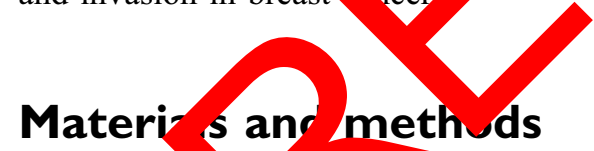

\section{Cell cultu. and tissue sample collection}

The MDA-MB-23 ell line was obtained from the Institute of Chemistry and Cell biology of the Chinese Academy of Sciences (Shanghai, China). These cells were cultured in DMEM supplemented with $10 \%$ fetal bovine serum.

The TNBC patient samples were collected from the Third Affiliated Hospital of Sun Yat-sen University. This experiment was approved by the Institutional Research Ethics Committee of Sun Yat-sen University. All patients provided written informed consent, and this experiment was conducted in accordance with the Declaration of Helsinki.

\section{RT-PCR analysis}

Total RNA was isolated from cells using Trizol reagent (Invitrogen). The first chain cDNA was synthesized using HiScript II Q-RT SuperMix for qPCR (+gDNA wiper, Vanzym, China). RT-PCR analyses were performed on a LightCycler 96 detection system (Roche) according to the manufacturer's instructions. All mRNA expression levels were normalized to the GAPDH signal.

\section{Production of lentivirus and cell infection} For the lentivirus-mediated suppre on LINC00511, the lentivirus containing LINCO0 1 shRNA re synthesized by Ruibo Co. Itd. Guangzhou China). A nontargeting scrambled nRNA $w_{c}$ also nerated as a negative control. Th shRN were crted into the pMKO.1-puro vectr. $\Delta$ ti al parti les were obtained by transfecting $3 \mathrm{~T}$ cells. Tiral pernatants were collected $72 \mathrm{hr}$ attc ransfection, or cell infection, MDAMB-231 cells were sod in six-well plates and infected with nitiviral particles xpressing LINC00511 shRNA and crambled s RNA. After $72 \mathrm{hrs}$, puromycin $(10 \mu \mathrm{g} /$ $\mathrm{mL}$, vas used to elect the puromycin-resistant clones.

\section{'nny formation and MTT assay}

pertorm colony formation assays, 200 cells were seeded in six-well culture plates and cultured for two eeks. After that, we washed the cells three times with PBS and stained them with Giemsa solution. Subsequently, the number of colonies containing $\geq 50$ cells was counted under a microscope. The plate clone formation efficiency was evaluated by using the formula: $=($ number of colonies $/$ number of cells inoculated $) \times 100 \%$.

An MTT assay was carried out as previously described. ${ }^{11}$ Briefly, cells were seeded into a 96-well plate and were allowed to grow for $24 \mathrm{hrs}$. Then, the media were aspirated, and MTT solution was added into each well. After incubation for 30 mins, $150 \mu \mathrm{L}$ DMSO was added into each well. Finally, the absorbance was read at $\mathrm{OD}=590 \mathrm{~nm}$.

\section{Cell invasion ability assay}

The cell invasion ability was examined with a Boyden assay. The cells were seeded into the upper chambers of the plate (Millipore), which were coated with $150 \mu \mathrm{g}$ Matrigel (BD Biosciences, Boston, MA, USA). Under the upper chambers, the lower chambers were filled with $500 \mu \mathrm{L}$ DMEM supplemented with $10 \%$ FBS. After incubation for $12 \mathrm{hrs}$, the cells adhering to the lower surface 
were fixed with methanol, stained with Giemsa solution and counted.

Flow cytometry assay

To carry out the cell-cycle assay, TNBC cells were harvested after $36 \mathrm{hrs}$ incubation and then washed with PBS. Subsequently, cells were fixed with $70 \%$ ice-cold ethanol at $4^{\circ} \mathrm{C}$ overnight, followed by incubation with propidium iodide and RNase A for 15 mins at $37^{\circ} \mathrm{C}$. After washed with cold PBS three times, FACS caliber flow cytometry (BD Biosciences) was used to gain the DNA content of labeled cells.

\section{Western blot assay}

Total proteins were extracted from cells with RIPA buffer (Beyotime, China) and were then separated on SDS-PAGE gels, followed by transfer to polyvinylidene fluoride (PVDF) membranes. We blocked the membranes with 3\% BSA/TBST and incubated them with primary antibodies at $4^{\circ} \mathrm{C}$ overnight. We then rinsed the PVDF membranes three times for 5 mins each with TBST and incubated the membranes in HRP-conjugated secondary antibodies for $1 \mathrm{hr}$ at room temperature. We detected the levels of total protein with enhanced chemiluminescence reagents.

\section{RNA pull-down assay}

Biotinylated RNA was synthesized in vi polymerase and then purified on G Sepha ex RN Columns (Roche). After validating the ize rit agm se gel electrophoresis, proper RN secondar tructure was folded from the biotin-label $R$. tion at different tempergyres. Mean hile, nuclear lysates were harvested from $\mathrm{ABC}$ cancer cell yith the addition of prewashed str avidin garose beads. A mixture of folded biotinylated $\mathrm{V}$ and near lysates were then sequentiall ino ated th $\mathrm{AA}$ and with prewashed streptay an-agar se beads, he pellets were washed and then bor r loam ouffer. The pulled-down BTRC protein was easured with Western blotting.

\section{RNA immunoprecipitation (RIP) assay}

Cells were grown in a $10 \mathrm{~cm}$ plate and were lysed in $0.5 \mathrm{~mL}$ RIP buffer (25 mM Tris $\mathrm{pH} 7.4,150 \mathrm{mM} \mathrm{KCl}$, $0.5 \mathrm{mM}$ DTT, and $0.5 \% \mathrm{NP}-40$ ) containing $100 \mathrm{U} / \mathrm{mL}$ RNase inhibitor and protease inhibitors and were centrifuged at 12,000 rpm for 10 mins. The supernatants were incubated separately with anti-Flag, anti-Slug, anti-mouse $\mathrm{IgG}$, or anti-rabbit $\mathrm{IgG}$ antibodies at $4^{\circ} \mathrm{C}$ for $2 \mathrm{hrs}$ with gentle rotation. Forty microliters of protein $\mathrm{A} / \mathrm{G}$ beads were added and incubated at $4^{\circ} \mathrm{C}$ for $1 \mathrm{hr}$. Beads were washed three times with RIP buffer and then once with PBS. RNA was extracted using Trizol, and RT-PCR was performed to analyze the samples.

\section{Tumor xenograft experiments}

Female BALB/c nude mice (five-weeks-old) were fed under standard conditions and cared for according to the institutional guidelines for animal care. The animal experiments were approved by the Instit Animal Care and Use Committee of Sun Yat-se niversity The sh-ctrl and sh-LINC00511 cells were inj subcutan pusly into the posterior flank of the nice. calcul the tumor volumes by using ane for vula ne $=$ length $\times$ width $^{2} / 2$ ). Five wo s af implantation, the xenografts were remoy from mice, ad the xenografts were weighed a Ki-67 assay was carried out to evaluate the pro eration index.

\section{results}

\section{INC005 I expression was upregulated} in $\mathrm{N}$ R tissues and was associated with worse prognosis

First, we used the TCGA database to examine the different lncRNAs in TNBC and normal breast tissues. A total of 1,030 lncRNAs were upregulated, while 369 lncRNAs were downregulated, in TNBC tissues when compared with those in normal breast tissues (fold change $>2$, Figure S1A and B). Among the elevated IncRNAs, LINC00511 was the most significantly different between the two tissues. Thus, we chose LINC00511 for further study.

Next, we analyzed the expression level of LINC00511 in a cohort of 87 TNBC patients. We found that the expression level of LINC00511 was upregulated in TNBC tissues compared with that in adjacent tissues (Figure 1A). In addition, we found that LINC00511 tended to be elevated in advanced-stage TNBC compared with that in early-stage TNBC patient samples (Figure 1B).

Subsequently, we examined whether LINC00511 was a noncoding RNA. Indeed, LINC00511 did not have coding capability, as revealed by an online bioinformatics analysis (http://cpc.cbi.pku.edu.cn/programs/run_cpc.jsp). An in vitro translation assay further confirmed that LINC00511 did not have coding capability (Figure S1C).

In addition, we explored the association between LINC00511 expression and the clinicopathological 
A

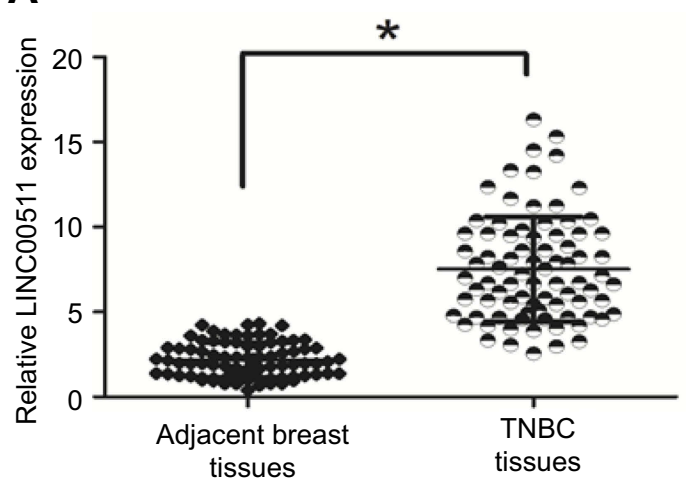

C

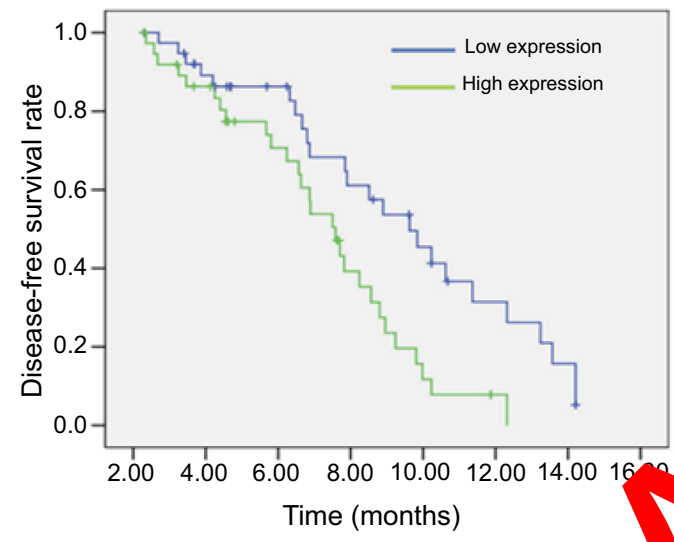

Figure I The LINC005II expression level was upregulated in TNP - icsues and upregulated in TNBC tissues compared with that in adjacent tis. (B) expre samples compared to those in normal breast tissues. (C) P ents with wer LIN LINC005II expression. (D) TNBC patients with higher le of LINCO L had shor

\section{B}

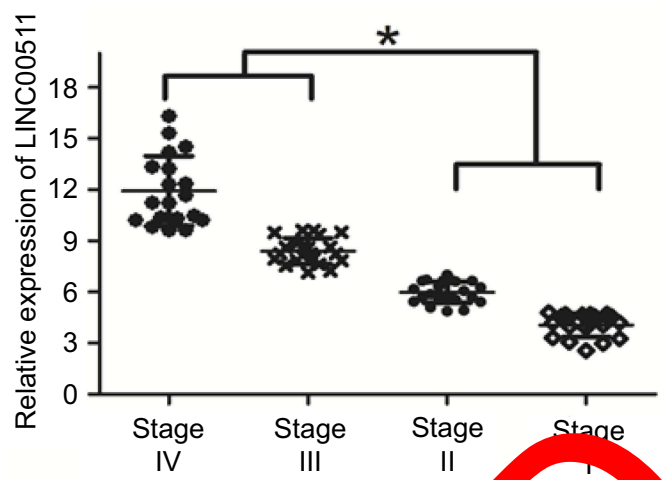

\section{D}

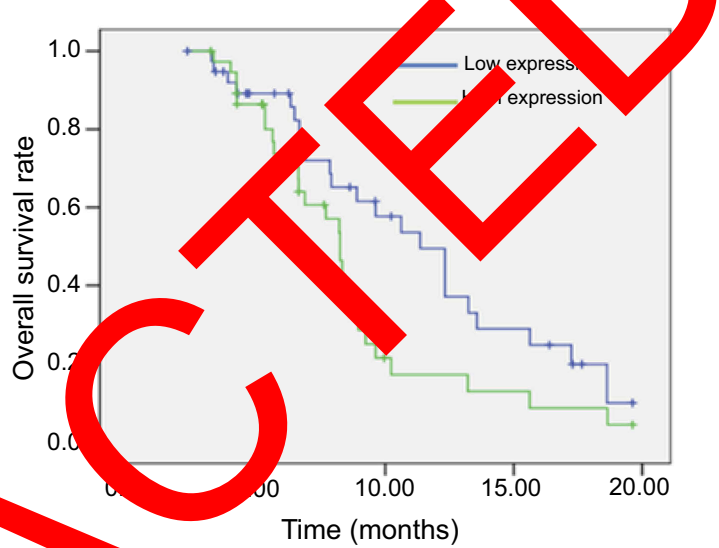

features of TNBC patients. As sho in Table elevated LINC00511 expression was si Aica as iv associato with tumor size $(P=0.000)$, lymp ${ }^{\prime}$ node meta sis $(P=0.014)$, and late clinical stage -0.007). Howeve, LINC00511 expression was not as rated age or smoking. TNBC patients with high expro levels LINC00511 had worse overall $\nabla_{10}$ ates a d ase-free survival rates than those natig is with $10 \mathrm{w}$ expression levels of LINC00511 GC anus).

\section{Downregulation of LINC005 I I inhibited MDA-MB-23 I cell growth and invasion}

These data suggested that a high expression level of LINC00511 was associated with tumor size and lymph node metastasis; thus, we examined whether LINC00511 affected TNBC cell growth and invasion. The MDA-MB-231 cell line was used to explore the biological role of LINC00511. First, we established MDA-MB-231 cells in which LINC00511 was stably knocked down (sh-LINC00511) (Figure 2A). An MTT assay revealed that LINC00511 downregulation decreased cell viability (Figure 2B) compared to that of the controls. A colony formation assay demonstrated that sh-LINC00511 cells formed smaller and fewer colonies than those formed by shctrl cells (Figure 2C). We subsequently asked whether LINC00511 affected the cell cycle distribution. Flow cytometry analysis demonstrated that MDA-MB-231 cells presented a significantly higher percentage of cells in the G1 phase and a lower percentage of cells in the $S$ phase in the sh-LINC00511 group compared with those in the sh-ctrl group (Figure 2D). The G1/S cell cycle checkpoint proteins (eg, cyclin D1, CDK4, and CDK6) were downregulated in sh-LINC00511 cells compared to those in the controls, as revealed by a Western blot assay (Figure 2E). We speculated that LINC00511 accelerated the cell cycle transition from the G1 phase to the $S$ phase and thus promoted MDA-MB-231 cell growth.

Subsequently, we examined whether LINC00511 affected the MDA-MB-231 cell invasion ability. A Boyden 
Table I Correlation between LINC005II expression and the clinicopathological profiles

\begin{tabular}{|c|c|c|c|c|}
\hline \multicolumn{2}{|c|}{$\begin{array}{l}\text { LINC005 I I } \\
\text { expression }\end{array}$} & \multirow{2}{*}{$\begin{array}{l}\text { Clinicopathological } \\
\text { profiles }\end{array}$} & \multirow[t]{2}{*}{$\mathbf{n}$} & \multirow[t]{2}{*}{$P$-value } \\
\hline High & Low & & & \\
\hline 25 & 21 & $<60$ & 46 & 0.456 \\
\hline 19 & 22 & $\geq 60$ & 41 & \\
\hline \multicolumn{5}{|c|}{ Smoking } \\
\hline 10 & 14 & Yes & 24 & 0.305 \\
\hline 34 & 29 & No & 63 & \\
\hline \multicolumn{5}{|c|}{ Tumor size } \\
\hline 33 & 16 & TI-2 & 49 & 0.000 \\
\hline II & 27 & T3-4 & 38 & \\
\hline \multicolumn{5}{|c|}{ Lymphatic invasion } \\
\hline 29 & 17 & Negative & 46 & 0.014 \\
\hline 15 & 26 & Positive & 43 & \\
\hline \multicolumn{5}{|c|}{ Distant metastasis } \\
\hline 12 & 24 & Yes & 36 & 0.007 \\
\hline 32 & 19 & No & 51 & \\
\hline
\end{tabular}

assay revealed that LINC00511 downregulation dec isea the cell invasion ability compared to that of the con lo (Figure 2F). It is well known that the EMT pnen ype ce tributes to cell invasion, ${ }^{12}$ and we the examin / whethe LINC00511 downregulation affecter th FN phenouge.

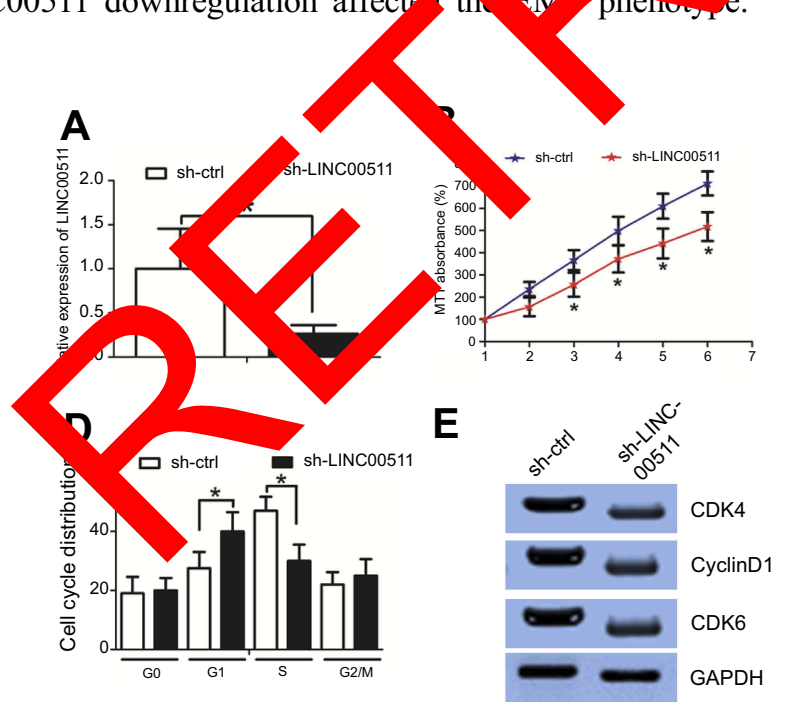

LINC00511 downregulation increased E-cadherin expression and decreased $\mathrm{N}$-cadherin and vimentin expression compared to those of the controls (Figure $2 \mathrm{G}$ ).

Taken together, these data revealed that the knockdown of LINC00511 decreased MDA-MB-231 cell growth and invasion.

\section{LINC005I I stabilized Snail by impeding Snail ubiquitination}

To explore the molecular mechanisms underlying the biological activity of LINC00511, we e ninea protein expression levels of key EMT ine ers (eg, Sna Slug, ZEB1, ZEB2, and Twist) in 2 -LIN 0511 an sh-ctrl cells. Interestingly, LINCO 11 downro latio decreased the Snail protein expre on ley (Figure - 1 ) compared to that in the controls owe $\mathrm{NCO05}^{1}$ downregulation did not affect the $1 \mathrm{mRNA}$ res level (data not shown). Snail is ghly stable, and stability of Snail is regulated by several ubiquit E3 ligases. ${ }^{13}$ To determine whether the RNA LINC00511 arects Snail stability in association with roteasome-r diated degradation, we treated cells with the oteasome i libitor MG132 to prevent Snail degradation. Co the of the controls, in the presence of both MG132 and the IncRNA LINC00511, the Snail protein levels incrused (Figure 3B), whereas these levels were significantly decreased following the lncRNA LINC00511 knockdown by siRNA in the absence of MG132 (Figure 3C). However, the addition of MG132 to LINC00511-depleted cells prevented
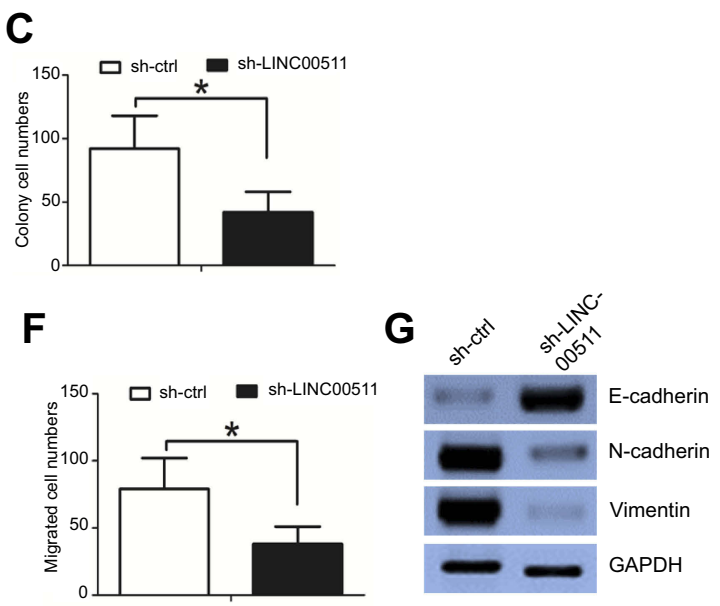

Figure 2 Downregulation of LINC005II inhibited MDA-MB-23I cell growth and invasion. (A) The transduction efficiency was validated by an RT-PCR assay. (B) An MTT assay revealed that LINC005II downregulation decreased cell viability compared to that of the controls. (C) A colony formation assay demonstrated that sh-LINC005II cells formed smaller and fewer colonies than those formed by sh-ctrl cells. (D) Flow cytometry analysis demonstrated that MDA-MB-23I cells presented a significantly higher percentage of cells in the GI phase and a lower percentage of cells in the S phase in the sh-LINC005II group than in the sh-ctrl group. (E) Western blot assays showed altered protein levels. (F) A Boyden assay revealed that LINC005II downregulation decreased cell invasion ability compared to that in the controls. (G) Western blot assays indicated that LINC005II downregulation increased E-cadherin expression and decreased $\mathrm{N}$-cadherin and Vimentin expression compared to those of the controls. ${ }^{*} p<0.05$. 

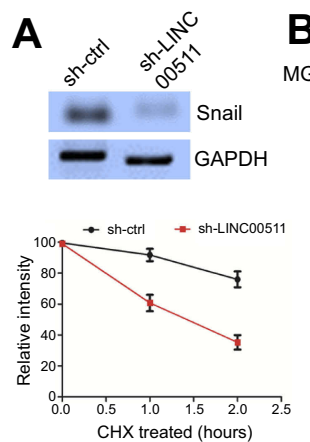

$\mathrm{CHX}$ treated (hours)
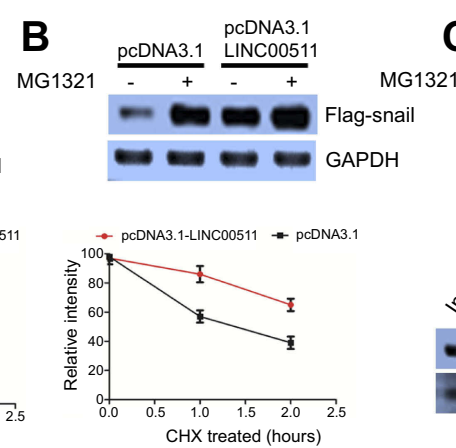
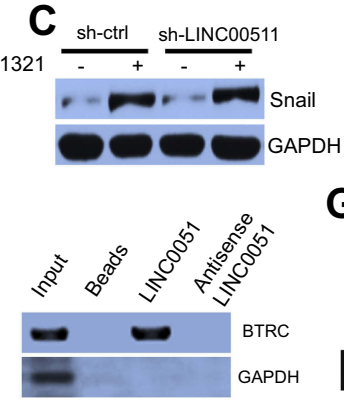

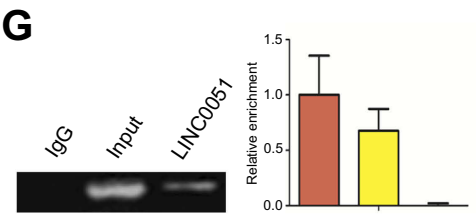

Input

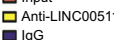

Figure 3 LINC005I I stabilizes Snail by impeding Snail ubiquitination. (A) LINC005I I downregulation decreased the Snail protein expression levalanmared to that of the control. (B) Snail protein levels increased in the presence of both MGI32 and IncRNA LINC005II compared to those in the controls. (C) nall pi levels decreased following IncRNA LINC005II knockdown by siRNA in the absence of MGI32 compared to those of the controls. (D) LINC005II down alation shorte the half-life of Snail compared to that of the controls. (E) LINC005II overexpression extended Snail half-life compared to that of the controls. (F) We vn blot assays rev that BTRC could be detected through its LINC005II interaction. (G) RIP assays indicated that BTRC is a LINC005II-associated protein.

the degradation of endogenous Snail compared to that of the controls (Figure 3C). These results suggested that LINC00511 is involved in the maintenance of Snail stability in a manner that is related to proteasomal degradation. Moreover, LINC00511 knockdown shortened the half-life (Figure 3D), whereas LINC00511 overexpression extended the Snail halflife, compared to that in the controls (Figure 3E).

To investigate the mechanisms associated with the LINC00511-mediated inhibition of Snail ubiquitination and identify the factors targeting Snail ubiquitination, we performe pull-down assays to screen for proteins that were motentially associated with LINC00511. RNA-protein comp' es the vere incubated with in vitro-transcribed biotiny ed-LIN 0511 and cell lysates were purified with cptav "nagnetic beads. The proteins in the LINC0051 ssociated po in complexes were then separated with $O \mathrm{~S}_{-} \mathrm{P}$ - $\mathrm{E}$, with tho corresponding antisense lncRNAs cting as the ntrols. Among these proteins, the $\beta$-tran cin repeat containit (BTRC) E3 ubiquitin protein ligase ss sele ed for further study (Figure S1D). To verify thrabovo entioned dings, we analyzed LINC00511 p -dowi proteî̀ wi Western blotting using a BTRC-sp fic anti dv WB revealed that BTRC could be detected amons oroteins that interacted with LINC00511 but not among the $\mathrm{p}_{-}$ins associated with either the antisense LINC00511 or with th beads alone (Figure 3F). Furthermore, to verify the interaction between LINC00511 and BTRC, we performed RIP assays for the RNA-protein complexes using BTRC-directed antibodies. Based on the qRT-PCR analysis, compared with the IgG or the anti-BTRC controls, the BTRCbound complexes showed a significant enrichment of LINC00511. No enrichment of LINC00511 was detected in the IgG complexes (Figure 3G). In summary, the data indicated that BTRC is a LINC00511-associated protein.
Subsequently, we $\mathrm{K}$ cked own the expression of the BTRC E3 ubiquit prote gase in DA-MB-231 cells, resulting in in sed Snail levels (Figure S1E). Consistent with finding, BTRC overexpression decrea ail protein vels in MDA-MB-231 cells compare to those in the controls. Furthermore, LINC00511 ove xpression i cued BTRC-induced Snail degradation (Fig S1F). Im ortantly, Snail ubiquitination was significantly a d by the overexpression of LINC00511 in 29. 1ls (Figure S1G), whereas the knockdown of endoonous LINC00511 in MDA-MB-231 cells increased the evels of endogenous Snail ubiquitination compared to ose of the controls (Figure S1H). These results indicated that LINC00511 blocks BTRC-mediated Snail ubiquitination by inhibiting the interaction between BTRC and Snail, thereby preventing Snail proteasomal degradation.

\section{Inhibition of LINC005I I decreased cell proliferation in vivo}

We further examined whether the inhibition of LINC00511 decreased cell proliferation in vivo. sh-LINC00511 or shctrl cells were inoculated into the back of the nude mice. Compared with the sh-ctrl cell-derived xenograft tumors, the sh-LINC00511 cell-derived xenograft tumors grew more slowly (Figure 4A). In addition, the mean weight of the sh-LINC00511 cell-derived xenograft tumors was lower than the sh-ctrl cell-derived xenograft tumors (Figure 4B). Interestingly, a Ki-67 staining assay also revealed that sh-LINC00511 cells had a lower proliferation index than sh-ctrl cells (Figure 4C). Taken together, these results suggested that the inhibition of LINC00511 decreased cell proliferation in vivo. 
A

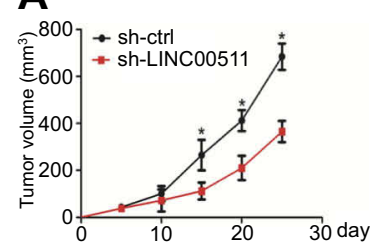

B

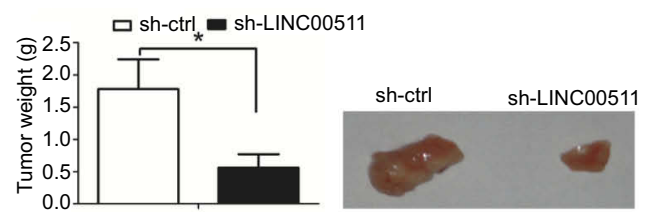

C

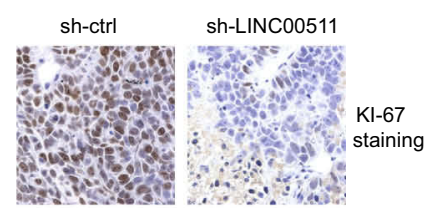

Figure 4 Inhibition of LINC005II decreased cell growth in vivo. (A) Compared with the sh-ctrl cell-derived xenograft tumors, the sh-LINC005II cell-derived xenograft tumors grew more slowly. (B) The mean weight of the sh-LINC005II cell-derived xenograft tumors was lower than that of the sh-ctrl cell-derived xenograft tumors. (C) A Ki-67 staining assay also revealed that sh-LINC005II cells had a lower proliferation index than that of sh-ctrl cells. ${ }^{*} p<0.05$.

\section{Discussion}

Recently, IncRNAs have been reported to influence breast tumorigenesis and progression via different mechanisms, such as regulating gene transcription, splicing, and binding to specific proteins. LncRNAs play a regulatory role in the coordination of protein molecules, so the identification of the specific protein targets of IncRNAs has become the main strategy for elucidating the lncRNA functions and mechanisms of action. In the present study, we performed a series of confirmatory experiments to demonstrate that LINC00511 is a lncRNA that associates with the Snail protein.

EMT is governed by a cohort of transcription factors, including members of the Snail, Zeb, and Twist families. The Snail protein is a core inducer of EMT. Snail directly i the transcription of E-cadherin and of several other inter lar adhesion components. The direct repress by Snail has been considered to be archet al of the ranscri tional regulation of EMT. In human Snail and E-cadherin is inversel correla and elevated levels of Snail are associated umor aggro veness and metastasis. Snail is highly unstabl and its stability has emerged as a key dete inant of the thelial phenotype. The stability of $\mathrm{Sn}$ is reg ated by several ubiquitin E3 ligases, including $\beta-\mathrm{p} 1 \quad$ oxl14/Fhx15, and Mdm2. ${ }^{13}$

In the study ve der nstrated that LINC00511 elevated Snail rotein o ossion level but did not affect the Sno mR sion level. We speculated that LINC0051 ay stabilize Snail by impeding Snail ubiquitination. Using R\ pull-down and RIP assays, we showed that LINC00511 interacted with the BTRC protein. BTRC encodes the $\beta \mathrm{TrCP}$ protein, a member of the F-box protein family and a key component of the SCF (Skp1-Cullin1-F-box)-type ubiquitin ligase E3. ${ }^{14}$ BTRC was also demonstrated to be an important factor in the process of EMT because of the $\beta \mathrm{TrCP}$ mediated ubiquitination of Snail in cancer, in which the inhibition of $\beta \mathrm{TrCP}$ resulted in the upregulation of Snail, which could induce EMT. ${ }^{15}$ We identified a novel mechanism associated with the regulation of Sno hility by demonstrating that LINC00511 binds to BT and blo Snail degradation. Our findings are similar previous do ments, which demonstrated that LINC 511 ack as an cogene during cancer progression.

In summary ou andi shed light on potential therapeutic strate s by tal fing the ncogenic IncRNA00511 in TNBC a ts.

\section{Cmlusion}

h summary our data revealed that the inhibition of INC00511 ecreased TNBC cell growth and invasion c nared those of the controls. Mechanistically, LINCous 1 maintained Snail stability by impeding its us itination and degradation via the BTRC E3 ubiquitin protein. We propose that the LINC00511/Snail axis may be a useful molecular target for the treatment of TNBC.

\section{Disclosure}

The authors report no conflicts of interest in this work.

\section{References}

1. Siegel RL, Miller KD, Jemal A. Cancer statistics, 2016. CA Cancer J Clin. 2016;66:7-30. doi:10.3322/caac.21332

2. Gupta GP, Massague J. Cancer metastasis: building a framework. Cell. 2006;127:679-695. doi:10.1016/j.cell.2006.11.001

3. Chiang AC, Massague J, Parish S, et al. Molecular basis of metastasis. $N$ Engl J Med. 2008;359:2814-2823. doi:10.1056/NEJMoa0801936

4. Lu L, Mao X, Shi P, et al. MicroRNAs in the prognosis of triple-negative breast cancer: a systematic review and meta-analysis. Medicine. 2017;96:e7085. doi:10.1097/MD.00000000 00007085

5. Turashvili G, Lightbody ED, Tyryshkin K, et al. Novel prognostic and predictive MicroRNA targets for triple-negative breast cancer .Faseb J. 2018. fj201800120R. doi:10.1096/fj.201800120R

6. Quinn JJ, Chang HY. Unique features of long non-coding RNA biogenesis and function. Nat Rev Genet. 2016;17:47-62. doi:10.1038/nrg.2015.10

7. Prensner JR, Chinnaiyan AM. The emergence of lncRNAs in cancer biology. Cancer Discov. 2011;1:391-407. doi:10.1158/2159-8290.CD11-0209 
8. Cano A, Perez-Moreno MA, Rodrigo I, et al. The transcription factor Snail controls epithelial-mesenchymal transitions by repressing E-cadherin expression. Nat Cell Biol. 2000;2:76-83. doi:10.1038/35000025

9. Blanco MJ, Moreno-Bueno G, Sarrio D, et al. Correlation of Snail expression with histological grade and lymph node status in breast carcinomas. Oncogene. 2002;21:3241-3246. doi:10.1038/sj.onc. 1205416

10. Muenst S, Daster S, Obermann EC, et al. Nuclear expression of Snail is an independent negative prognostic factor in human breast cancer. Dis Markers. 2013;35:337-344. doi:10.1155/2013/902042

11. Deng X, Ma L, Wu M, et al. miR-124 radiosensitizes human glioma cells by targeting CDK4. J Neurooncol. 2013;114:263-274. doi:10.1007/ s11060-013-1179-2

12. Kong P, Chen L, Yu M, et al. miR-3178 inhibits cell proliferation and metastasis by targeting Notch1 in triple-negative breast cancer. Cell Death Dis. 2018;9:1059. doi:10.1038/s41419-018-1091-y

13. Diaz VM, Vinas-Castells R, Garcia de Herreros A. Regulation of the protein stability of EMT transcription factors. Cell Adhes Migration. 2014;8:418-428. doi:10.4161/19336918.2014.969998
14. He N, Li C, Zhang X, et al. Regulation of lung cancer cell growth and invasiveness by beta-TRCP. Mol Carcinog. 2005;42:18-28. doi:10.1002/ (ISSN) 1098-2744

15. Zhou BP, Deng J, Xia W, et al. Dual regulation of Snail by GSK-3betamediated phosphorylation in control of epithelial-mesenchymal transition. Nat Cell Biol. 2004;6:931-940. doi:10.1038/ncb1173

16. Lu G, Li Y, Ma Y, et al. Long noncoding RNA LINC00511 contributes to breast cancer tumourigenesis and stemness by inducing the miR-185-3p/E2F1/Nanog axis. J Exp Clin Cancer Res. 2018;37:289. doi:10.1186/s13046-018-0945-6

17. Xu S, Kong D, Chen Q, Ping Y, Pang D. Oncogenic long noncoding RNA landscape in breast cancer. Mol Cancer. 2017;16:129. doi:10.1186/ s12943-017-0696-6

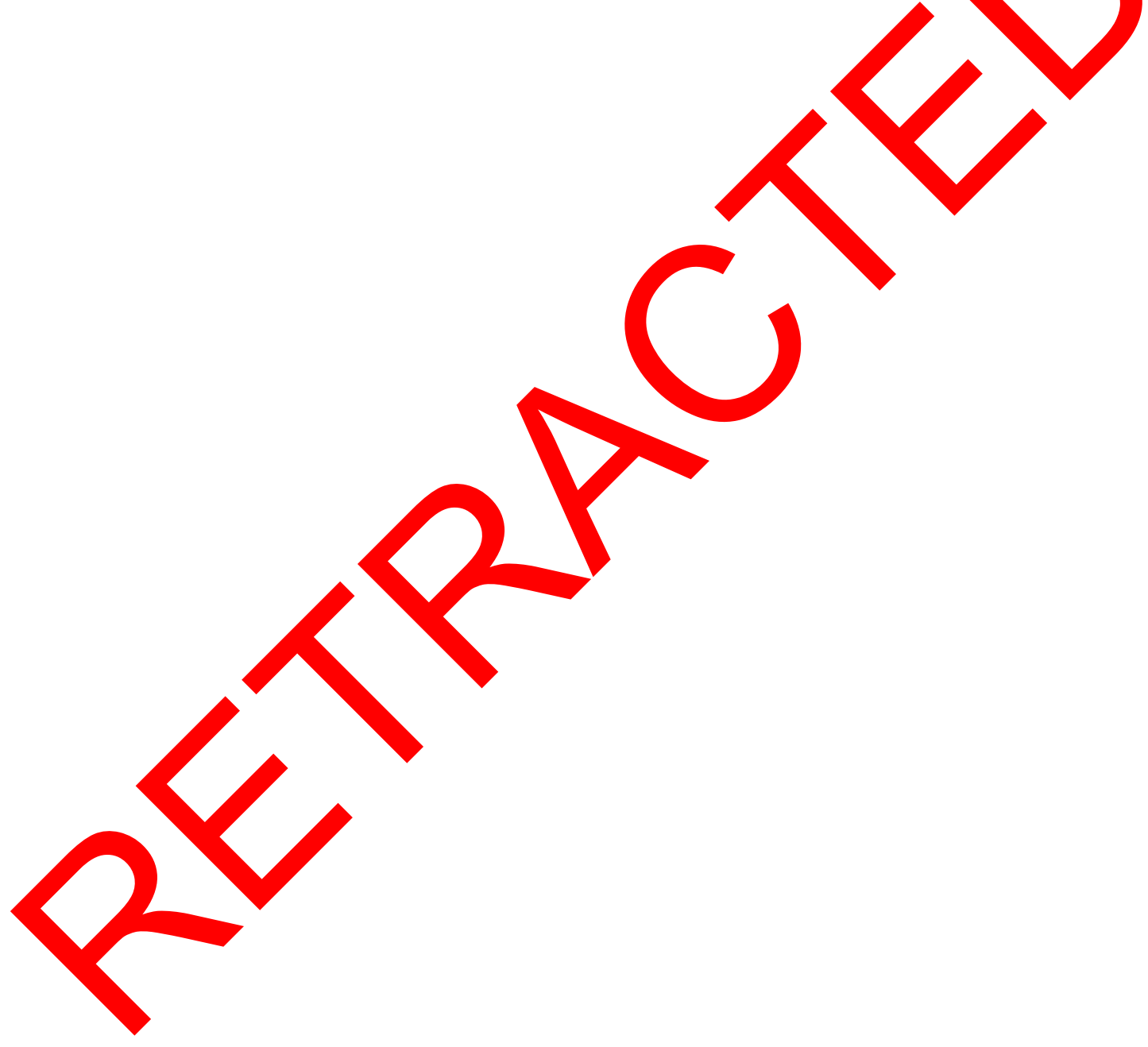




\section{Supplementary Material}

A

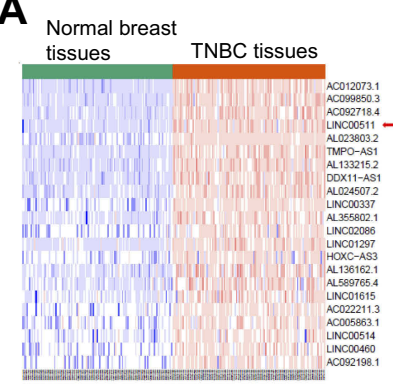

C

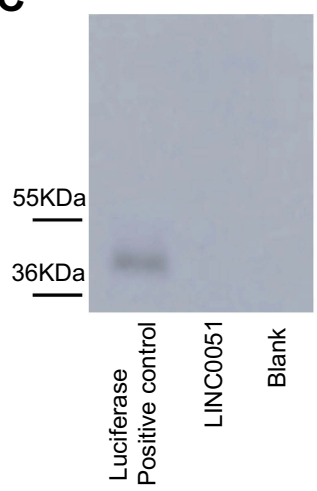

B

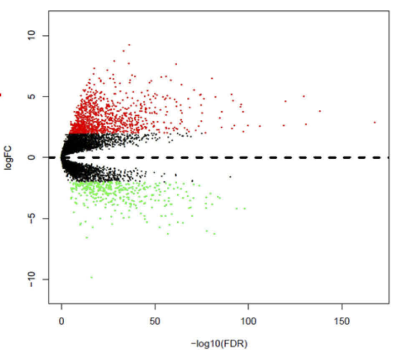

D

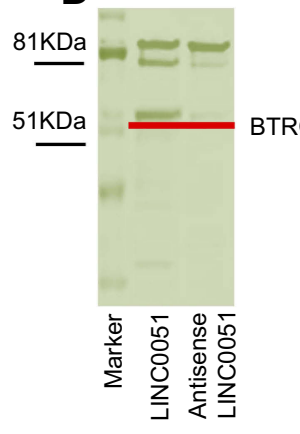

\section{E}

G

G

HA-UB FLAG-snail LINC00511

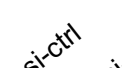

م<smiles>[CH]=[CH]</smiles>
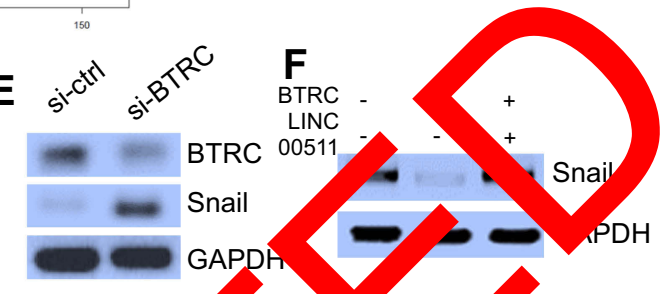

BTR

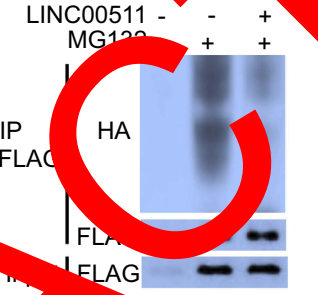

H

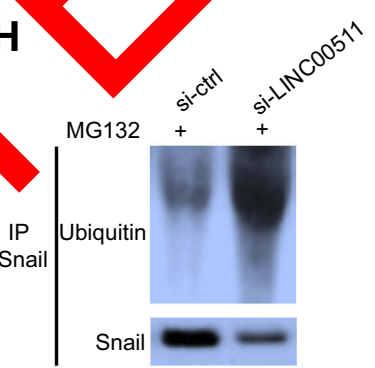

Figure SI (A) and (B) The downregulated and upregulated In between the he and normal tissues in the TCGA database, as shown by the heat map and plot. (C) An in vitro translation assay further confirmed th $\triangle N$ NCO I did no ave protein-coding capability. (D) Identification of the LINC005II-protein complex with an incubation of biotinylated-LINC005II with protein 6 acts by cc nassie blu aining assay. The $\beta$-transducin repeat containing (BTRC) E3 ubiquitin protein ligase was therefore selected as the candidate protein. (E) BT E3 ubiqu downregulation resulted in increased Snail protein levels compared to those of the controls. (F) BTRC overexpression decreased S.I pro le in MDA-ThI cells compared to those of the controls. LINC005II overexpression rescued BTRCinduced Snail degradation. (G) and (H) 293T IDA-MB-2 ells were transfected with the indicated vectors and treated with MGI32 for 6 hrs. Then, cell lysates were immunoprecipitated using an antibody agai tag $(\mathbf{G})$ or $\mathrm{HA}$ body $(\mathbf{H})$. The precipitates and inputs were analyzed by immunoblotting.

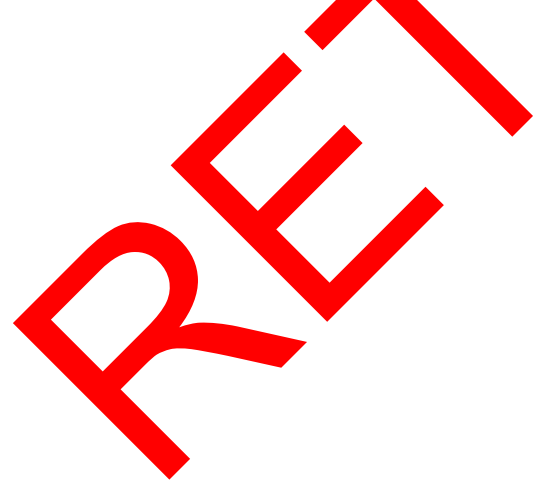

Cancer Management and Research

Dovepress

\section{Publish your work in this journal}

Cancer Management and Research is an international, peer-reviewed open access journal focusing on cancer research and the optimal use of preventative and integrated treatment interventions to achieve improved outcomes, enhanced survival and quality of life for the cancer patient.
The manuscript management system is completely online and includes a very quick and fair peer-review system, which is all easy to use. Visit http://www.dovepress.com/testimonials.php to read real quotes from published authors. 